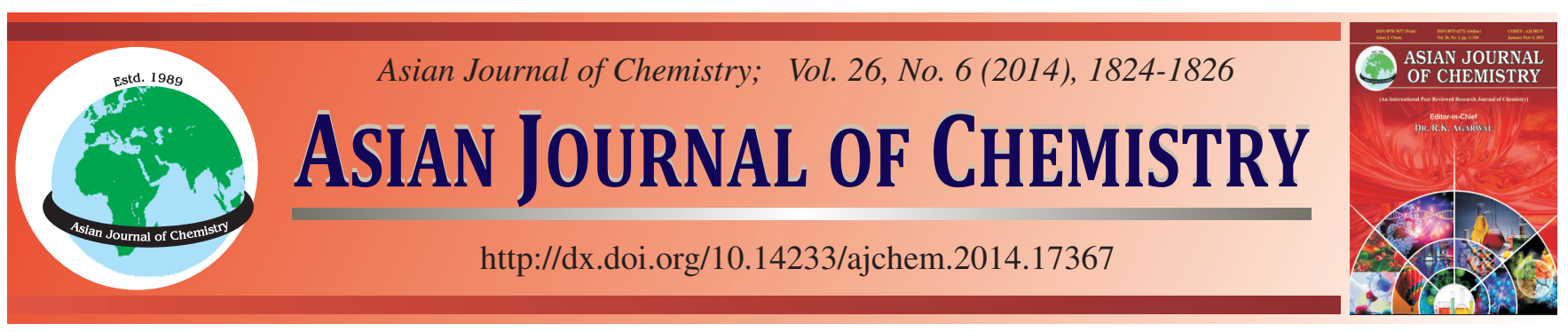

\title{
Selective Synthesis of Bismuth Nanoflower by L-Aspartic Acid Assisted Hydrothermal Method $\uparrow$
}

\author{
Jinsong XiE ${ }^{1,2, *}$, Yucheng Guan ${ }^{1}$, Changan Tian ${ }^{1,2}$, Hongdian LU ${ }^{1,2}$, \\ Chengliang Han ${ }^{1,2}$, Qiyi Yin ${ }^{1,2}$, Difang ZhaO ${ }^{1,2}$ and Ming Ding ${ }^{1,2}$
}

\begin{abstract}
${ }^{1}$ Department of Chemistry and Materials Engineering, Hefei University, Hefei 230601, Anhui Province, P.R. China ${ }^{2}$ Key Lab of Powder and Energy Sources Materials, Hefei University, Hefei 230601, Anhui Province, P.R. China

*Corresponding author: E-mail: xjs153@hfuu.edu.cn
\end{abstract}

\begin{abstract}
In this paper, three dimensional (3D) flower-like bismuth nanostructures constructed by nanorods were synthesized by a hydrothermal method, using L-aspartic acid and bismuth nitrate as raw materials and deionized water as solvent. The morphologies, structures of asprepared products were tested by X-ray diffraction, field emission scanning electron microscopy, transmission electron microscopy, respectively. The optimized reaction time, ratio of reactants and the $\mathrm{pH}$ value of the solution were given by different experimental conditions. The effects of reaction conditions on the morphologies of the products were investigated and the formed mechanism was also premilarily discussed.
\end{abstract}

Keywords: 3D bismuth nanoflower, Hydrothermal synthesis, Morphology conversion.

\section{INTRODUCTION}

Nowadays, as a kind of semimetal, bismuth materials with micro and nano-structures have attracted much attention owing to their unusual thermoelectric properties ${ }^{1}$, large magneto resistance ${ }^{2}$, etc. In order to investigate the change of properties aroused from the varying of the morphology of bismuth, various bismuth materials, including one-dimensional structures ${ }^{3}$ of nanotubes ${ }^{4,5}$, nanowires ${ }^{6}$, nanorods ${ }^{7}$, nanobelts ${ }^{8}$; zero-dimensional structures of nanoparticles ${ }^{9,10}$, nanospheres ${ }^{11}$, hollow nanospheres ${ }^{12}$, nanocubes ${ }^{13}$ and three dimensional structures of nanosheets ${ }^{14}$, bunch-like nanostructures ${ }^{15}$, have been prepared by using diverse methodologies, such as electrodeposition $^{6,15}$, solvothermal method ${ }^{12}$, chemical reduction route $^{14,16}$, thermal decomposition ${ }^{9}$, refluxing method ${ }^{11}$, etc. Up to now, there are few reports on synthesis of 3D bismuth nanoflower formed by 1D nanorod. Herein, an easy L-aspartic acid induced hydrothermal synthesis strategy was introduced to prepare uniform $3 \mathrm{D}$ bismuth nanoflowers. Importantly, the synthesized Bi nanoflowers can been changed into $(\mathrm{BiO})_{2} \mathrm{CO}_{3}$ nanoplates by varying the reaction temperature and time, which was not reported previously. The obtained $\mathrm{Bi}$ and $(\mathrm{BiO})_{2} \mathrm{CO}_{3}$ nanomaterials have potential significant applications for electrical field and environment remediation.

\section{EXPERIMENTAL}

All chemicals were of analytical grade reagents and used without further purification. A typical case as follows: $1 \mathrm{mmol}$ of $\mathrm{Bi}\left(\mathrm{NO}_{3}\right)_{3} \cdot 5 \mathrm{H}_{2} \mathrm{O}$ and $2.5 \mathrm{mmol} \mathrm{L}$-aspartic acid were dissolved into $15 \mathrm{~mL}$ deionized water and $5 \mathrm{~mL}$ of $1 \mathrm{M} \mathrm{NaOH}$. The mixture was stirred for $15 \mathrm{~min}$ and then ultrasonic treatment for $15 \mathrm{~min}$ at room temperature. Finally, the mixture was put into a Teflon-lined stainless steel autoclave with a capacity of $25 \mathrm{~mL}$. The autoclave was maintained in a digital-type temperature-controlled oven at $140^{\circ} \mathrm{C}$ for $6 \mathrm{~h}$. As the autoclave cooled to room temperature naturally and then, the as-obtained black products were centrifuged, washed respectively with absolute ethanol and deionized water several times and dried at $60{ }^{\circ} \mathrm{C}$ for $5 \mathrm{~h}$. By varying the molar ratio of the reactant, the $\mathrm{pH}$ value of the solvents and the reaction temperature and time; the obtained products were labeled by S1-S4 as shown in Table-1.

$\mathrm{X}$-ray diffraction pattern of samples were examined on a Bruker D8-advance X-ray diffractometer with $\mathrm{CuK}_{\alpha}$ radiation $(\lambda=1.54056 \AA$ ). FE-SEM images were measured on a Hitachi S-4800 field emission scanning electron microanalyser employing an operating voltage of $10 \mathrm{kV}$. TEM images and high-

†resented at The 7th International Conference on Multi-functional Materials and Applications, held on 22-24 November 2013, Anhui University of Science \& Technology, Huainan, Anhui Province, P.R. China 


\begin{tabular}{|c|c|c|c|c|c|c|}
\hline & MORF & $\begin{array}{l}\text { YNTH } \\
\text { HOLO }\end{array}$ & $\begin{array}{l}\text { TAF } \\
\text { ESIS PA } \\
\text { IIES OF }\end{array}$ & $\begin{array}{l}\text { LE-1 } \\
\text { AMET } \\
\text { HE PR }\end{array}$ & $\begin{array}{l}\text { RS ANI } \\
\text { DUCT }\end{array}$ & $1-S 4$ \\
\hline Sample & $\begin{array}{c}\text { Molar } \\
\text { ratio }\end{array}$ & $\begin{array}{l}\mathrm{H}^{+} / \\
\mathrm{OH}^{-}\end{array}$ & $\begin{array}{c}\text { Temp. } \\
\left({ }^{\circ} \mathrm{C}\right)\end{array}$ & $\begin{array}{l}\text { Time } \\
\text { (h) }\end{array}$ & Color & Morphology \\
\hline S1 & $1: 2.5$ & - & 180 & 10 & White & \\
\hline S2 & $1: 5$ & - & 180 & 10 & Gray & \\
\hline S3 & $1: 2.5$ & $\mathrm{OH}^{-}$ & 140 & 6 & Black & \\
\hline S4 & $1: 2.5$ & $\mathrm{H}^{+}$ & 140 & 6 & White & \\
\hline
\end{tabular}

resolution transmission electron microscopy (HRTEM) image were carried out on a JEM-2100F transmission electron microscope.

\section{RESULTS AND DISCUSSION}

The typical XRD pattern of the prepared 3D bismuth nanoflowers is displayed (Fig. 1). All of the reflection peaks of the XRD pattern in Fig. 1 can be well indexed to be hexagonal rhomb-centered phase of bismuth structure (JCPDS card No. 05-0519). No other peak appeared in the XRD pattern, which showed that the obtained products have pure phase. Additionally, the sharp and narrow XRD peaks indicated that the prepared 3D Bi nanoflowers have good crystalline structure. In order to investigate the detailed morphologies and structures of the samples, the obtained products were further characterized by FE-SEM, TEM images and XRD patterns as given in Figs. 2 and 3. A large number of the bismuth nanoflowers structure with uniform sizes can be easily observed in Fig. $2 \mathrm{a}$, most of which have average sizes $2 \mu \mathrm{m}$. The magnified FE-SEM and the TEM images confirmed that the bismuth

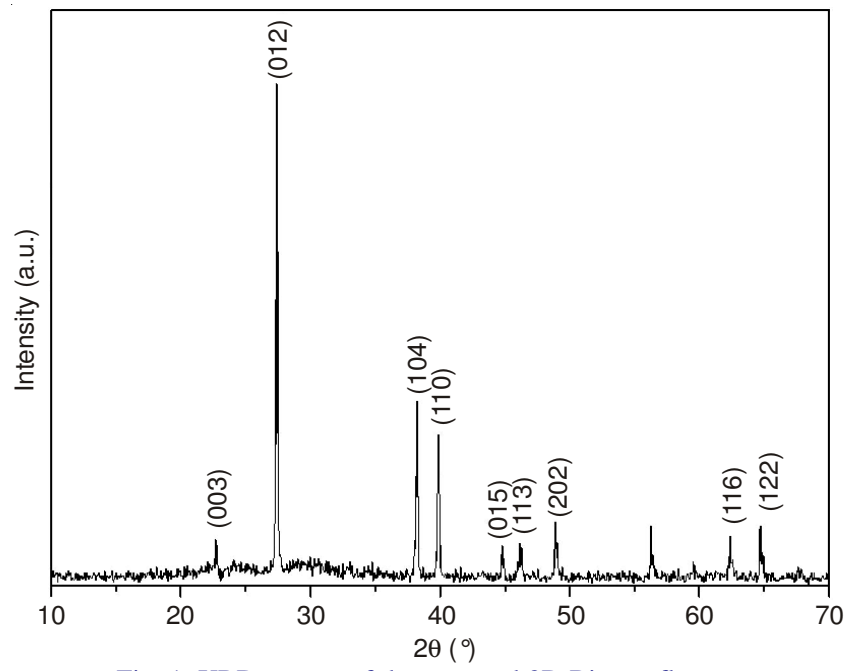

Fig. 1. XRD pattern of the prepared 3D Bi nanoflowers nanoflowers exhibited irradiative flower-like structure and were composed by many tiny nanorods with the diameter of $100 \mathrm{~nm}$ as shown in Fig. 2b-c. The as-obtained 3D bismuth nanoflowers have good crystalline structures as illustrated in Fig. 2d.
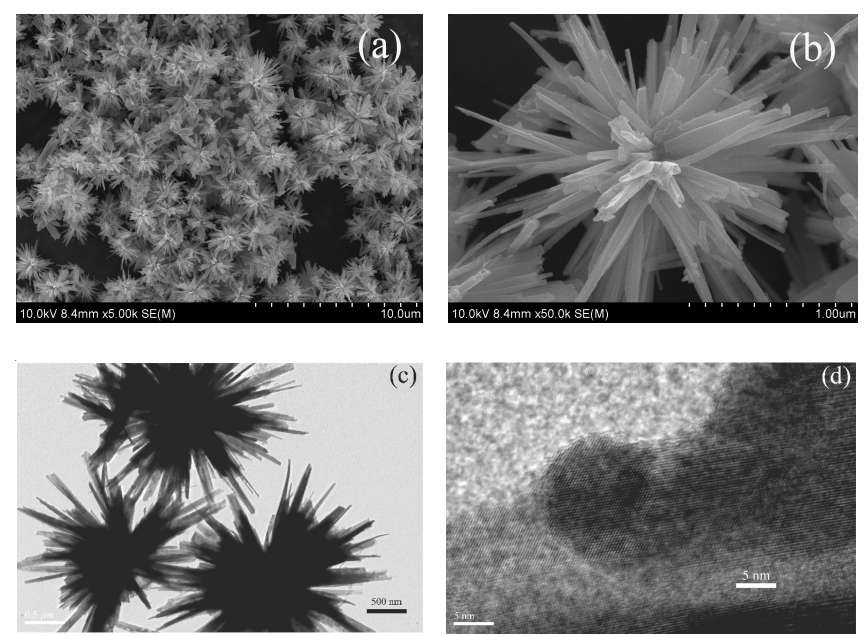

Fig. 2. Low magnification (a), high magnification FE-SEM images (b), TEM (c) and HRTEM (d) images of as-obtained 3D bismuth nanoflowers

To further investigate the influences of experimental conditions on the structures of the obtained products, we carried out a series of experiments by varying the parameters of the experiments shown in Table-1. When no $\mathrm{NaOH}$ in the reaction system, the products can turn into $(\mathrm{BiO})_{2} \mathrm{CO}_{3}$ nanoplates with the increasing the reaction time and temperature to $10 \mathrm{~h}$ and $180{ }^{\circ} \mathrm{C}$, respectively. Keeping the other condition constant, the products can also transfer into $(\mathrm{BiO})_{2} \mathrm{CO}_{3}$ nanoplates when the reaction $\mathrm{NaOH}$ was been changed into nitric acid. Based on the above observations, we pointed out that the $\mathrm{pH}$ value and the amount of L-aspartic acid were the main important factors of the formation of 3D bismuth nanoflowers. Herein, the added L-aspartic acid not only acts as a solvent but also as a reducing and morphology directing agent in this synthetic process. There are some similar function reagents, such as ethylene glycol (EG) in the synthesis of bismuth hollow nanospheres ${ }^{12}$, glycine in the formation of 1D $\mathrm{LaOHCO}_{3}$ nanostrctures ${ }^{17}$. However, the accurate formation mechanism is still require further study.

\section{Conclusion}

An L-aspartic acid assisted hydrothermal method was developed to obtain large-scale bismuth nanoflowers with uniform morphology. The experimental results indicated the reaction temperature, the $\mathrm{pH}$ value and the $\mathrm{L}$-aspartic acid play critical role in the formation of $\mathrm{Bi}$ nanoflowers. It is believed that this amino acid-induced method can become a universal route to manufacture other different metallic nanostructures, such as $\mathrm{Cu}, \mathrm{Fe}, \mathrm{Ag}$, etc.

\section{ACKNOWLEDGEMENTS}

The authors thank the financial supports from the National Natural Science Foundation of China (No. 51102073, 51276054), the Natural Science Foundation of Anhui Province 

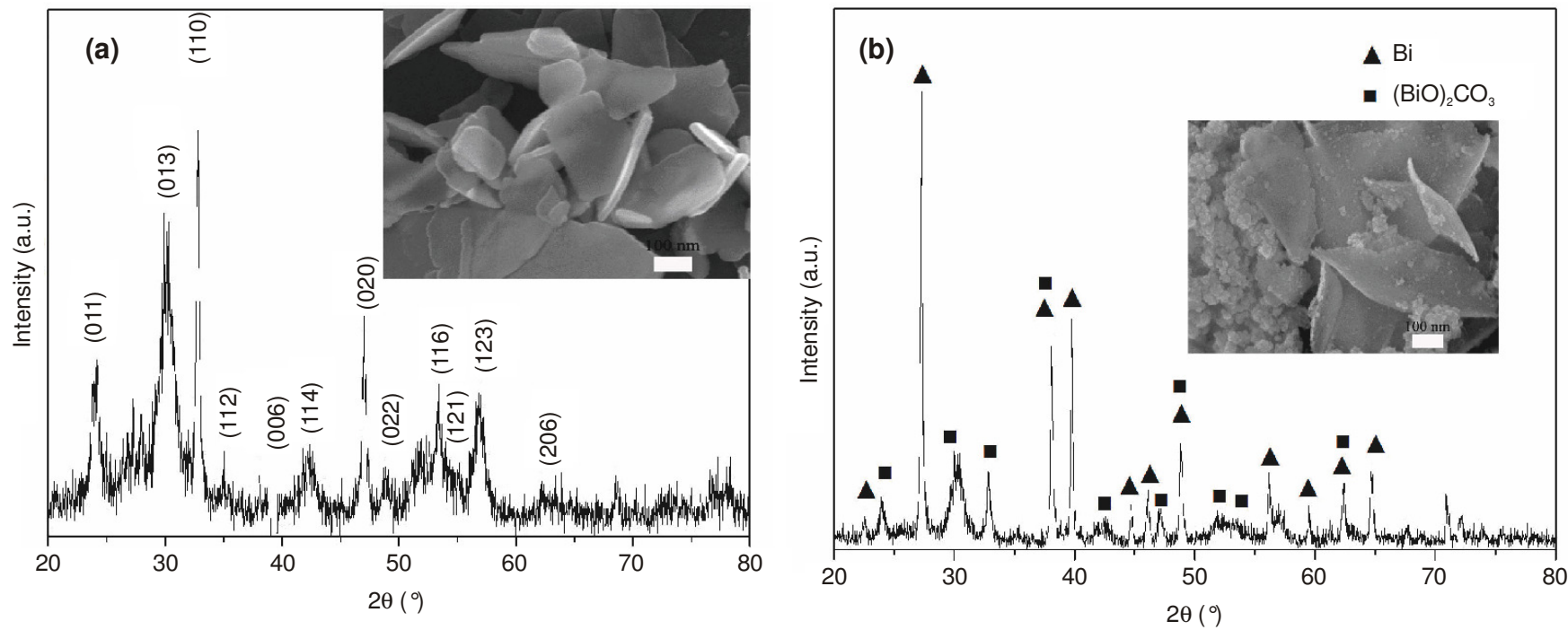

Fig. 3. XRD patterns and FE-SEM images of sample S1 (a) and S2 (b)

(No. 1308085QB35), the Natural Science Foundation of Education Department of Anhui Province (No. KJ2012B154, KJ2012B148, KJ2013B229), the Research Fund of Key Laboratory for Advanced Technology in Environmental Protection of Jiangsu Province(AE201361) and the College Students' Innovation and Entrepreneurship Training Program of China (No. 201211059006, 201211059007, 201211059011, 201211059009, 201211059005).

\section{REFERENCES}

1. J.S. Son, K. Park, M.K. Han, C. Kang, S.G. Park, J.H. Kim, W. Kim, S.J. Kim and T. Hyeon, Angew. Chem. Int. Ed., 50, 1363 (2011).

2. F.Y. Yang, K. Liu, K.M. Hong, D.H. Reich, P.C. Searson and C.L. Chien, Science, 284, 1335 (1999).

3. D.C. Ma, J.Z. Zhao, Y. Zhao, X.L. Hao and Y. Lu, Chem. Eng. J., 209, 273 (2012).

4. Y.D. Li, J.W. Wang, Z.X. Deng, Y.Y. Wu, X.M. Sun, D.P. Yu and P.D. Yang, J. Am. Chem. Soc., 123, 9904 (2001).

5. R. Boldt, M. Kaiser, D. Köhler, F. Krumeich and M. Ruck, Nano Lett., 10, 208 (2010)
6. Y.G. Zhu, X.C. Dou, X.H. Huang, L. Li and G.H. Li, J. Phys. Chem. B, 110, 26189 (2006).

7. Y.W. Wang, J.S. Kim, J.Y. Lee, G.H. Kim and K.S. Kim, Chem. Mater., 19, 3912 (2007).

8. Y.T. Chen, R. Gong, W. Zhang, X.Q. Xu, Y.Q. Fan and W.S. Liu, Mater. Lett., 59, 909 (2005).

9. G. Carotenuto, C.L. Hison, F. Capezzuto, M. Palomba, P. Perlo and P. Conte, J. Nanopart. Res., 11, 1729 (2009).

10. X. Chen, S. Chen, W. Huang, J.F. Zheng and Z.L. Li, Electrochim. Acta, 54, 7370 (2009).

11. G. Cheng, J.L. Wu, F. Xiao, H. Yu, Z. Lu, X.L. Yu and R. Chen, Mater. Lett., 63, 2239 (2009).

12. F. Qin, G.F. Li, H. Xiao, Z. Lu, H.Z. Sun and R. Chen, Dalton Trans., 41, 11263 (2012).

13. W.Z. Wang, B. Poudel, Y. Ma and Z.F. Ren, J. Phys. Chem. B, 110, 25702 (2006).

14. P. Kumar, J. Singh and A.C. Pandey, RSC Adv., 3, 2313 (2013).

15. Z. Zhang, K. Yu, D. Bai and Z.Q. Zhu, Nanoscale Res. Lett., 5, 398 (2010).

16. S. Derrouiche, C.Z. Loebick and L. Pfefferle, J. Phys. Chem. C, 114, 3431 (2010).

17. J.S. Xie, Q.S. Wu, D. Zhang and Y.P. Ding, Cryst. Growth Des., 9, 3889 (2009). 